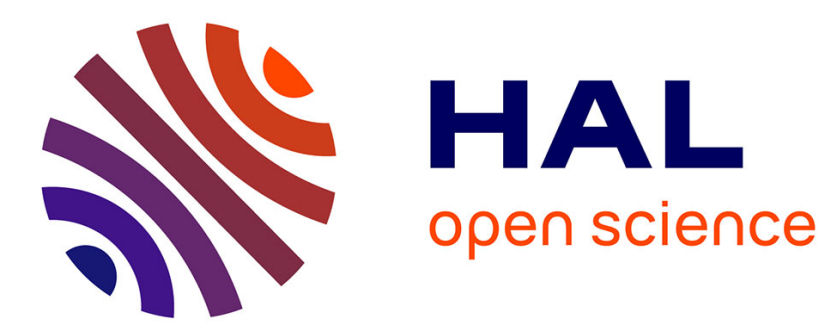

\title{
Observation of the Earth's nutation by the VLBI: how accurate is the geophysical signal
}

César Gattano, Sébastien Lambert, Christian Bizouard

\section{To cite this version:}

César Gattano, Sébastien Lambert, Christian Bizouard. Observation of the Earth's nutation by the VLBI: how accurate is the geophysical signal. Journal of Geodesy, 2016. hal-01376213

\section{HAL Id: hal-01376213 \\ https://hal.science/hal-01376213}

Submitted on 4 Oct 2016

HAL is a multi-disciplinary open access archive for the deposit and dissemination of scientific research documents, whether they are published or not. The documents may come from teaching and research institutions in France or abroad, or from public or private research centers.
L'archive ouverte pluridisciplinaire HAL, est destinée au dépôt et à la diffusion de documents scientifiques de niveau recherche, publiés ou non, émanant des établissements d'enseignement et de recherche français ou étrangers, des laboratoires publics ou privés. 


\title{
- Observation of the Earth's nutation by the VLBI: how accurate is the geophysical signal
}

\author{
César Gattano • Sébastien B. Lambert . \\ 4 Christian Bizouard
}

5

6 Received: date / Accepted: date

\begin{abstract}
We compare nutation time series determined by several International VLBI Service for geodesy and astrometry (IVS) analysis centers. These series were made available through the International Earth Rotation and Reference Systems Service (IERS). We adjust the amplitudes of the main nutations, including the free motion associated with the free core nutation (FCN). Then, we discuss the results in terms of physics of the Earth's interior. We find consistent FCN signals in all of the time series, and we provide corrections to IAU 2000A series for a number of nutation terms with realistic errors. It appears that the analysis configuration or the software packages used by each analysis center introduce an error comparable to the amplitude of the prominent corrections. We show that the inconsistencies between series have significant consequences on our understanding of the Earth's deep interior, especially for the free inner core resonance: they induce an uncertainty on the FCN period of about 0.5 day, and on the free inner core nutation (FICN) period of more than 1000 days, comparable to the estimated period itself. Though the FCN parameters are not so much affected, a 100\% error shows up for the FICN parameters and prevents from geophysical conclusions.
\end{abstract}

Keywords VLBI · Earth Rotation · Nutation · Free Core Nutation (FCN) · Free Inner Core Nutation (FICN)

César Gattano

SYRTE, Observatoire de Paris, PSL Research University, CNRS, Sorbonne Universités, UPMC Univ. Paris 06, LNE

E-mail: cesar.gattano@obspm.fr

Sébastien B. Lambert

SYRTE, Observatoire de Paris, PSL Research University, CNRS, Sorbonne Universités, UPMC Univ. Paris 06, LNE

E-mail: sebastien.lambert@obspm.fr

Christian Bizouard

SYRTE, Observatoire de Paris, PSL Research University, CNRS, Sorbonne Universités, UPMC Univ. Paris 06, LNE

E-mail: christian.bizouard@obspm.fr 


\section{Introduction}

Very long baseline radio interferometry (VLBI; Shaffer, 1995) is the only geodetic technique able to measure the nutation of the Earth. The accuracy of the order of 0.1 millisecond of arc (mas) allows researchers to compare the observed nutation with theoretical prediction model for a rigid Earth and, henceforth, constrain geophysical parameters describing the Earth's interior (e.g., Gwinn et al, 1986; Herring et al, 1986, 1991, 2002; Mathews et al, 1991, 1995, 2002; Koot et al, 2008, 2010). Inaccuracies in nutation measurements must therefore be taken into account when developing an Earth model. The identification and the elimination of sources of error in VLBI analysis become crucial for the search of the tiny signature of the free inner core nutation whose resonant period is not clearly determined (Mathews et al, 2002; Koot et al, 2008, 2010) and its excited free motion is thought to be of amplitude smaller than 0.001 mas (Dehant et al, 2005), and thus undetectable in VLBI observations.

Since the 1980s, almost 6000 VLBI 24-h sessions have been scheduled by various space geodesy agencies for monitoring the Earth's rotation as regularly as possible and to determine accurate terrestrial and celestial reference frames. This large amount of data (consisting of about 10 million delays spanning about 35 years) is regularly reanalyzed by several research teams in the framework of VLBI analysis centers (ACs) affiliated to the International VLBI Service for Geodesy and Astrometry (IVS; Nothnagel et al, 2015) and the International Earth Rotation and Reference Systems Service (IERS).

One problem arising with nutation is that one cannot objectively know if one series is more precise than another, i.e., if it better describes the physical phenomenon it is supposed to describe. The reason is that there is no basis of comparison like, e.g., an independent technique or an accurate theoretical predicting model. One can only compare nutation series to other nutation series obtained with a very similar analysis chain. Actually, time series show significant differences at the level of a few tens of microseconds of arc ( $\mu$ as) originating from analysis options or software packages (Tesmer et al, 2007; Heinkelmann and Tesmer, 2013; Gattano et al, 2015a,b). Answering the question which series is the best for geophysical research is an open question, except if some ACs use outdated modeling or evidently bad data analysis procedures.

In this paper, we propose to assess the consistency of nutation time series made available by the IERS. Beyond the statistical overview, we investigate the geophysical signal present in all of the series and made up of mismodeled or unpredictable nutations (Section 2). Section 3 is dedicated to discussing the impact of the inconsistencies on deep Earth interior parameters, namely the resonant frequencies associated with the core and the inner core.

\section{Data analysis}

We considered solutions provided by nine IVS ACs plus three combinations (Fig. 1). They are listed in Table 1 . All individual series are publicly available and documented at the IVS data centers ${ }^{1}$ or at the institution web sites. The IVS combination is based on a weighted average of pre-reduced normal equations (Böckmann

\footnotetext{
$1 \mathrm{ftp}: / /$ ivscc.gsfc.nasa.gov/pub/vlbi
} 
et al, 2007). The IERS EOP 08 C 04 produced at the IERS Earth Orientation Center is based on a smoothing of the IVS combination, interpolated to a 1-day interval and densified by some individual series (Bizouard and Gambis, 2009). Bulletin A delivered by the IERS Rapid Service/Prediction Center is built up from combination of both IVS combination and individual series.

The precise identification of the causes of the differences between series is out of the scope of this paper: such an identification cannot be done by a comparison of a posteriori solutions but would need extensive tests and comparisons between software packages. We therefore do not detail the strategies adopted by each AC, but we refer the reader to the technical documents mentioned above. Nevertheless, one must keep in mind that there are several prominent sources of error limiting the accuracy of VLBI nutation series. First, one must consider software issues including the inversion method: though Calc/Solve and VieVS (Böhm et al, 2012) use classical weighted least-squares, OCCAM (Titov et al, 2004) implements Kalman filtering. The software packages differ in to what level state-of-the-art modelling (Petit and Luzum, 2010) is realized. Second, the number of processed sessions, and thereby the number of observations that constrain the global parameters like radio source or station coordinates, varies drastically from one series to another. Although most of the IVS ACs are now processing as many sessions as possible, the possible bad influence of regional networks or sessions not designed for EOP measurement should be addressed. Third, the instability of the targeted radio sources can generate a noise in EOP of a few tens of $\mu$ as (Dehant et al, 2003; FeisselVernier, 2003; Feissel-Vernier et al, 2005, 2006). The adequate analysis strategy to minimize this noise is still an open question. For instance, although most of the IVS ACs now use the ICRF2 (Fey et al, 2015), some operational solutions are still based on ICRF1 (Ma et al, 1998), whose accuracy and stability are worse by factors of 5 and 2, respectively. To finish with, an important factor limiting the accuracy of VLBI products is the station-dependent correlated noise associated with clocks and troposphere modeling errors in absence of covariance error model (Gipson, 2007; Romero-Wolf et al, 2012). As the number of observations increases, the correlated noise becomes dominant with respect to the thermal noise that tends to zero on average. The impact of this effect partly depends on the troposphere wet delay, gradient, and clock offsets estimation intervals, as well as the choice of the mapping functions (Böhm et al, 2006).

Most of time series contain offsets to the IAU 2000A nutation model, based on the model of Mathews et al (2002), also referred to as MHB in the following. These offsets of about 0.2 mas in rms are attributed to various unmodeled or mismodeled geophysical effects (Dehant et al, 2003) as well as a noise-like signal due to imperfections of the analysis strategy. The majority of the series are also referred to the IAU 2006 precession (Capitaine et al, 2003). We corrected series based on older references so that all our series represent differences to the precession-nutation model consisting of the IAU 2006 precession and the IAU 2000A nutation.

We modeled the series by (i) a set of corrections to the main nutation amplitudes (circular terms with known astronomical frequencies and phases and unknown constant amplitudes), (ii) corrections to precession rate and constant biases due to a misorientation of the celestial reference frame, and (iii) a retrograde circular term accounting for the free core nutation (FCN) whose variability and unpredictability is discussed in several studies on the light of the various possible excitation sources accounted for by different existing atmospheric and oceanic 
models as well as triggering by geomagnetic jerks (Herring et al, 2002; Shirai et al, 2005; Lambert, 2006; Vondrak and Ron, 2009; Malkin, 2013; Vondrák and Ron, 2016). For item (i), we chose the 21 prograde and retrograde nutations used by the MHB authors to fit their geophysical model. For (iii), the adjustment of the FCN amplitude was done using a running window. The FCN period was fixed to the MHB value ( -430.21 days). The window width was set to 6.7 years in order to ensure the demodulation of the FCN and the annual retrograde nutation. We therefore have an overlap of data so that the annual values of the FCN amplitude are not independent. However, the overlap allows us to record interannual amplitude variations and constitutes a good compromise between time and frequency resolution. The obtained FCN amplitudes (Fig. 2) are all consistent within error bars and comparable to those raised by other studies (Herring et al, 2002; Lambert, 2006; Malkin, 2007; Vondrak and Ron, 2009; Krásná et al, 2013; Chao and Hsieh, 2015; Belda et al, 2016). The FCN amplitudes adjusted by Malkin (2007) are shown in the figure. They appear globally consistent with ours. Deviations can be explained by the different adjustment strategy: Malkin (2007) used a different input series (smoothed IVS combined series) and adjusted the FCN amplitude on a 400-d interval running by step of one day. Note that we do not show the comparison with the IERS model (Petit and Luzum, 2010) since the derivation method is extremely close to our scheme and the results do not exhibit noticeable differences.

Our fitting method is based on least-squares with weights taken as the inverse of the squared errors given in the series. These errors are derived from least-squares estimation propagated from delay weights and constraints applied at various stages of the analysis to source and station positions, clocks and troposphere parameters. Delay weights have been defined in a preliminary analysis (not processed by us but by special IVS ACs) as a combination of a formal uncertainty of the ionosphere group delays at $\mathrm{X}$ and S-band and a station-dependent uncertainty determined such that the chi-squared of the residuals within each session is close to unity. For each VLBI session, the error associated with nutation is therefore consistent with the standard deviation of delay residuals. However, the fit of the above model to time series leaves reasonably flat residuals with $\chi^{2}$ significantly larger than unity, suggesting that errors are underestimated. Because they use a larger number of observations, large network sessions can produce small errors. However, there exists systematics between sessions due to, e.g., changing network or target source geometry. For white noise residuals, these discrepancies should be explained by the errors, so that the rms of the scaled residuals (or, equivalently, the $\chi^{2}$ ) should be close to unity, which is not the case. To remedy this inconsistency, we included an iterative recalibration of errors by a scale factor $s$ and an error floor $f$ in our fitting algorithm so that our final estimates achieve (i) a minimum sum of squared differences between observations and model and (ii) a standard deviation of the residuals consistent with the errors (Herring et al, 1991, 2002). The recalibrated error $\sigma_{\text {rec }}$ is such that $\sigma_{\text {rec }}^{2}=(s \sigma)^{2}+f^{2}$.

Corrections to precession rate and bias are displayed in Table 2. Formal uncertainties are typically 0.005 mas for bias and 0.05 mas/yr for precession rate. Although most of precession rates are in agreement within one sigma, five series present significantly different values. The reason is unclear but should be investigated because it could lead to misinterpretation of the measured precession rate in terms of Earth's flattening. 
Error floors and scale factors are displayed in Table 2 with the postfit rms (i.e., the signal unexplained by corrected nutations and FCN). Our analysis yields an averaged error floor of 0.074 mas, an averaged scale factor of 1.47 , and an averaged median error of 0.157 mas. There is no clear dependence on the software package although the solutions obtained with Calc/Solve tend to show a slightly smaller scale factor: on average, the scale factor for Calc/Solve solutions is 1.42, while it is 1.83 for other software packages. However, this tendency is not true for noise floors and median errors. One important remark arising from Table 2 is that the IVS combined series present the smallest postfit rms, scale factor and median error. This indicates that the combined series is actually more accurate than the individual contributions, and therefore more reliable for geophysical studies.

\section{Discussion}

The observed corrections to IAU 2000A nutation amplitudes are displayed in Table 3. Though corrections were obtained independently for each series, the table does not display all the results but only the median amplitudes of the individual corrections, the highest range (difference between the maximum and minimum values), and median errors. Doing so, we aim at pointing out the differences between amplitudes obtained from different series rather than the amplitudes themselves.

Corrections relevant to most important nutations for geophysical applications are displayed in the upper panels of Fig. 3. The retrograde 18.6-yr nutation shows the highest range and standard deviation. This was expected since, in addition to the fact that the observing time only integrates two periods, this term is strongly influenced by the first decade of VLBI observations when VLBI sessions were not as dense as nowadays and realized through weaker networks. The impact of such differences on further estimates of geophysical parameters can be evaluated by considering the frequency-domain MHB transfer function linking the rigid Earth nutation (REN) series (Souchay et al, 1999) to non-rigid series. After adding up the values of Table 3 to the corresponding IAU 2000A amplitudes, we removed the nonlinear and sun-synchronous contributions of Table 7 of the MHB paper and derived the ratio to the REN series. Then, we adjusted the values of the FCN and FICN complex frequencies (see, e.g., Eq. (42) of MHB). We used MHB values as a priori parameters and followed a classical nonlinear iterative least-squares scheme that converges after a few iterations (Mathews et al, 2002).

Whisker plots of FCN and FICN periods and quality factors are shown in the lower panels of Fig. 3 where the real period $P$ and the damping factor $Q$ are such that the complex resonant frequency $\sigma$ reads $(1 / P)(1-\mathrm{i} / 2 Q)$. Solution iaa2007a returned an unexpectedly high FICN period close to 9000 days with a similarly higher uncertainty. Nevertheless, we arranged the scale relevant to this parameter so that one can better appreciate the differences between other solutions. We found that the FCN period stays within one half day with uncertainties of $\sim 0.2$ day. The quality factor remains roughly between 16000 and 18000, in agreement with Rosat and Lambert (2009) obtained by a similar method, with uncertainties between 600 and 900. For both the FCN period and the quality factor, the dispersion of the results associated with the $\mathrm{AC}$ is of the order of the median uncertainty, so that one can reasonably claim that the analysis strategy does not significantly perturb the estimates of this geophysical parameter. The situation is different for the FICN. 
Even excluding iaa2007a, the FICN period ranges an interval of the order of the period itself ( $\sim 1300$ days $)$, indicating that this parameter is particularly sensitive to the analysis strategy.

\section{Conclusion}

In this study, we analyzed various available nutation series provided by different analysis in terms of nutation amplitude. The results emphasizes that the analysis strategy does not have a significant influence on the estimates of the FCN parameters but does have one on the FICN parameters. Such a large dispersion can make the detection of the FICN by VLBI questionable. It is therefore urgent to investigate thoroughly the sources of error in VLBI analysis, especially for deriving nutation offsets that give important insight into the Earth's interior. It is clear that providing as many series as possible with different softwares and strategies is useful for the scientific community only if the submission of the series to the international services (IVS, IERS) is followed by a rigorous assessment of their quality for scientific use. The assessment method has still to be discussed, as well as the combination schemes that are supposed to wipe out the defects of individual data sets and return series optimized for scientific exploitation.

\section{References}

Belda S, Ferrándiz JM, Heinkelmann R, Nilsson T, Schuh H (2016) Testing a new free core nutation empirical model. Journal of Geodynamics 94:59-67

Bizouard C, Gambis D (2009) The combined solution c04 for earth orientation parameters consistent with international terrestrial reference frame 2005. In: Geodetic reference frames, Springer, pp 265-270

Böckmann S, Artz T, Nothnagel A, Tesmer V (2007) Comparison and combination of consistent vlbi solutions. In: Proceedings of the 18th European VLBI for geodesy and astrometry working meeting, 79, pp 82-87

Böhm J, Werl B, Schuh H (2006) Troposphere mapping functions for gps and very long baseline interferometry from european centre for medium-range weather forecasts operational analysis data. Journal of Geophysical Research: Solid Earth 111(B2)

Böhm J, Böhm S, Nilsson T, Pany A, Plank L, Spicakova H, Teke K, Schuh H (2012) The new vienna vlbi software vievs. In: Geodesy for Planet Earth, Springer, pp 1007-1011

Capitaine N, Wallace PT, Chapront J (2003) Expressions for iau 2000 precession quantities. Astronomy \& Astrophysics 412(2):567-586

Chao BF, Hsieh Y (2015) The earth's free core nutation: Formulation of dynamics and estimation of eigenperiod from the very-long-baseline interferometry data. Earth and Planetary Science Letters 432:483-492

Dehant V, Feissel-Vernier M, de Viron O, Ma C, Yseboodt M, Bizouard C (2003) Remaining error sources in the nutation at the submilliarc second level. Journal of Geophysical Research: Solid Earth 108(B5) 
Dehant V, De Viron O, Greff-Lefftz M (2005) Atmospheric and oceanic excitation of the rotation of a three-layer earth. Astronomy \& Astrophysics 438(3):11491161

Feissel-Vernier M (2003) Selecting stable extragalactic compact radio sources from the permanent astrogeodetic vlbi program. Astronomy \& Astrophysics 403(1):105-110

Feissel-Vernier M, Ma C, Gontier AM, Barache C (2005) Sidereal orientation of the earth and stability of the vlbi celestial reference frame. Astronomy \& Astrophysics 438(3):1141-1148

Feissel-Vernier M, Ma C, Gontier AM, Barache C (2006) Analysis strategy issues for the maintenance of the icrf axes. Astronomy \& Astrophysics 452(3):11071112

Fey A, Gordon D, Jacobs C, Ma C, Gaume R, Arias E, Bianco G, Boboltz D, Böckmann S, Bolotin S, et al (2015) The second realization of the international celestial reference frame by very long baseline interferometry. The Astronomical Journal 150(2):58

Gattano C, Lambert S, Bizouard C (2015a) Comparison of official IVS nutation time series from VLBI analysis. In: Martins F, Boissier S, Buat V, Cambrésy L, Petit P (eds) SF2A-2015: Proceedings of the Annual meeting of the French Society of Astronomy and Astrophysics, pp 115-119

Gattano C, Lambert S, Bizouard C, Souchay J (2015b) Studying impacts of strategy choices concerning the Celestial Reference Frame on the estimates of nutation time series during geodesic VLBI Analysis. IAU General Assembly $22: 2252261$

Gipson J (2007) Incorporating correlated station dependent noise improves vlbi estimates. In: Proc. 18th European VLBI for Geodesy and Astrometry Working Meeting, Vienna, Austria, pp 12-13

Gwinn CR, Herring TA, Shapiro II (1986) Geodesy by radio interferometry: Studies of the forced nutations of the earth: 2. interpretation. Journal of Geophysical Research: Solid Earth 91(B5):4755-4765

Heinkelmann R, Tesmer V (2013) Systematic inconsistencies between vlbi crf and trf solutions caused by different analysis options. In: Reference Frames for Applications in Geosciences, Springer, pp 181-189

Herring T, Gwinn C, Shapiro I (1986) Geodesy by radio interferometry: Studies of the forced nutations of the earth: 1. data analysis. Journal of Geophysical Research: Solid Earth 91(B5):4745-4754

Herring T, Mathews P, Buffett B (2002) Modeling of nutation-precession: Very long baseline interferometry results. Journal of Geophysical Research: Solid Earth 107(B4)

Herring TA, Buffett BA, Mathews P, Shapiro II (1991) Forced nutations of the earth: Influence of inner core dynamics: 3. very long interferometry data analysis. Journal of Geophysical Research: Solid Earth 96(B5):8259-8273

Koot L, Rivoldini A, De Viron O, Dehant V (2008) Estimation of earth interior parameters from a bayesian inversion of very long baseline interferometry nutation time series. Journal of Geophysical Research: Solid Earth 113(B8)

Koot L, Dumberry M, Rivoldini A, De Viron O, Dehant V (2010) Constraints on the coupling at the core-mantle and inner core boundaries inferred from nutation observations. Geophysical Journal International 182(3):1279-1294 
Krásná H, Böhm J, Schuh H (2013) Free core nutation observed by vlbi. Astronomy \& Astrophysics 555:A29

Lambert S (2006) Atmospheric excitation of the earth's free core nutation. Astronomy \& Astrophysics 457(2):717-720

Ma C, Arias E, Eubanks T, Fey A, Gontier AM, Jacobs C, Sovers O, Archinal B, Charlot P (1998) The international celestial reference frame as realized by very long baseline interferometry. The Astronomical Journal 116(1):516

Malkin Z (2007) Empiric models of the earths free core nutation. Solar System Research 41(6):492-497

Malkin Z (2013) Free core nutation and geomagnetic jerks. Journal of Geodynamics 72:53-58

Mathews P, Buffett BA, Herring TA, Shapiro II (1991) Forced nutations of the earth: Influence of inner core dynamics: 1. theory. Journal of Geophysical Research: Solid Earth 96(B5):8219-8242

Mathews P, Buffett B, Shapiro I (1995) Love numbers for a rotating spheroidal earth new definitions and numerical values. Geophysical research letters 22(5):579-582

Mathews PM, Herring TA, Buffett BA (2002) Modeling of nutation and precession: new nutation series for nonrigid earth and insights into the earth's interior. Journal of Geophysical Research: Solid Earth 107(B4)

Nothnagel A, Alef W, Amagai J, Andersen PH, Andreeva T, Artz T, Bachmann S, Barache C, Baudry A, Bauernfeind E, et al (2015) The IVS data input to ITRF2014. In: GFZ Data Services, Helmoltz Centre, Potsdam, Germany, DOI 10.5880/GFZ.1.1.2015.002

Petit G, Luzum B (2010) Iers conventions (2010). Tech. rep., DTIC Document

Romero-Wolf A, Jacobs CS, Ratcliff JT (2012) Effects of Tropospheric Spatiotemporal Correlated Noise on the Analysis of Space Geodetic Data. In: Behrend D, Baver KD (eds) Seventh General Meeting (GM2012) of the international VLBI Service for Geodesy and Astrometry (IVS), held in Madrid, Spain, March 4-9, 2012, Eds: D. Behrend and K.D. Baver, National Aeronautics and Space Administration, p. 231-235, pp 231-235

Rosat S, Lambert S (2009) Free core nutation resonance parameters from vlbi and superconducting gravimeter data. Astronomy \& Astrophysics 503(1):287-291

Shaffer DB (1995) Geodesy. In: Zensus JA, Diamond PJ, Napier PJ (eds) Very Long Baseline Interferometry and the VLBA, Astronomical Society of the Pacific Conference Series, vol 82, p 345

Shirai T, Fukushima T, Malkin Z (2005) Detection of phase disturbances of free core nutation of the earth and their concurrence with geomagnetic jerks. Earth, planets and space 57(2):151-155

Souchay J, Loysel B, Kinoshita H, Folgueira M (1999) Corrections and new developments in rigid earth nutation theory-iii. final tables. Astronomy and Astrophysics Supplement Series 135(1):111-131

Tesmer V, Boehm J, Heinkelmann R, Schuh H (2007) Effect of different tropospheric mapping functions on the trf, crf and position time-series estimated from vlbi. Journal of Geodesy 81(6-8):409-421

Titov O, Tesmer V, Boehm J (2004) OCCAM v.6.0 Software for VLBI Data Analysis. In: Vandenberg NR, Baver KD (eds) International VLBI Service for Geodesy and Astrometry 2004 General Meeting Proceedings, p 267 
Vondrak J, Ron C (2009) Stability of period and quality factor of free core nutation. Acta Geodyn Geomater 6(3):217-224

Vondrák J, Ron C (2016) Geophysical fluids from different data sources, geomagnetic jerks, and their impact on Earth s orientation. Acta Geodyn
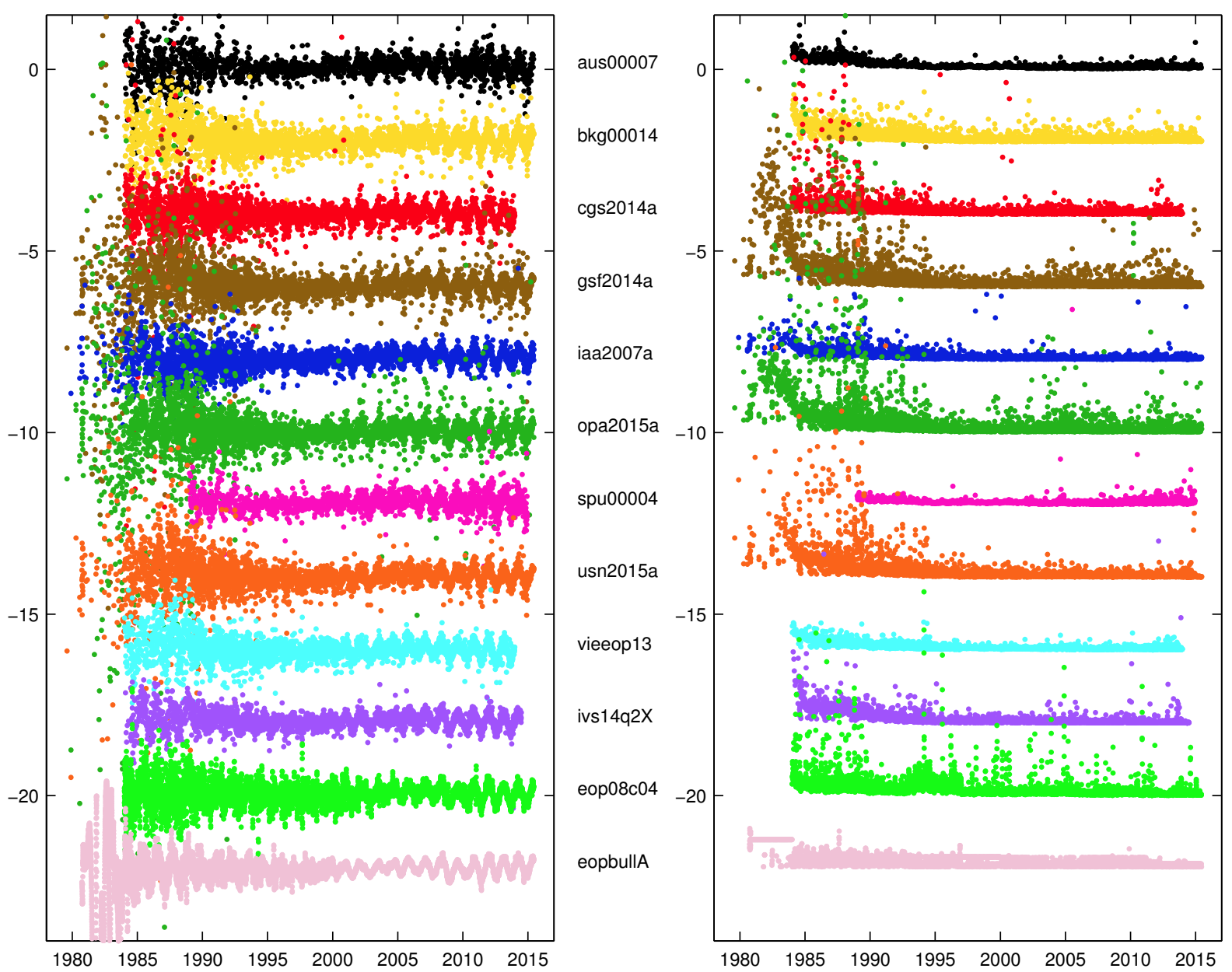

Fig. 1 (Left) Nutation series available from the IVS and the IERS and (Right) their respective errors. Y-axes are labelled in mas. For clarity purposes, the series have been shifted by steps of 2 mas and only the $X$-component is represented. The $Y$-component shows a similar behavior. 
Table 1 Identifiers of the nutation time series corresponding to the IVS solution code (except for eop08c04 and eopbullA which name have been chosen by the authors), hosting institute of the IVS ACs, number of processed sessions (i.e., length of the series), and time span.

\begin{tabular}{|c|c|c|c|c|}
\hline & Analysis center \& Software package & & Sessions & Span \\
\hline aus00007 & Geoscience Australia, Canberra & OCCAM & 2469 & $1984.0-2015.4$ \\
\hline bkg00014 & Bundesamt für Kartographie und Geodäsie, Leipzig, Germany & Calc/Solve & 4615 & $1984.0-2015.4$ \\
\hline $\operatorname{cgs} 2014 \mathrm{a}$ & Centro di Geodesia Spaziale, Matera, Italy & Calc/Solve & 4743 & $1984.0-2013.9$ \\
\hline gsf2014a & NASA Goddard Space Flight Center (GSFC), Greenbelt, MD & Calc/Solve & 5360 & $1979.5-2015.4$ \\
\hline iaa2007a & Institute of Applied Astronomy, Saint Petersburg, Russia & OCCAM & 4391 & $1979.9-2015.4$ \\
\hline opa2015a & Paris Observatory, France & Calc/Solve & 5671 & $1979.5-2015.4$ \\
\hline spu00004 & Saint-Petersburg University, Russia & OCCAM & 2067 & $1989.0-2014.9$ \\
\hline usn2015a & United States Naval Observatory (USNO), Washington, DC & Calc/Solve & 4842 & $1979.5-2015.4$ \\
\hline vieeop13 & Vienna University of Technologies, Austria & VieVS & 3768 & $1984.0-2014.0$ \\
\hline ivs $14 \mathrm{q} 2 \mathrm{X}$ & IVS Combination Center, Germany & - & 3403 & $1984.0-2014.4$ \\
\hline eop08c04 & IERS Earth Orientation Center, Paris Observatory, France & - & 11487 & $1983.9-2015.4$ \\
\hline eopbullA & IERS Rapid Service/Prediction Center, Washington, DC & - & 12681 & $1980.7-2015.4$ \\
\hline
\end{tabular}

Table 2 Bias $b$ (mas) and corrections to precession rate $p$ (mas/yr), postfit rms $r$ (mas) of the residuals after the fit of the annual values of the FCN amplitude and the corrections to IAU 2000A nutation amplitudes, noise floor $f$ (mas), scale factor $s$, and median error $m$ (mas) of the series.

\begin{tabular}{lrrrrrrrrr}
\hline \hline & $b_{X}$ & $b_{Y}$ & $p_{X}$ & $p_{Y}$ & $r_{X}$ & $r_{Y}$ & $f$ & $s$ & $m$ \\
\hline aus00007 & 0.068 & -0.111 & 0.152 & -0.512 & 0.159 & 0.162 & 0.066 & 2.00 & 0.173 \\
bkg00014 & 0.029 & -0.060 & 0.149 & -0.103 & 0.146 & 0.145 & 0.083 & 1.46 & 0.151 \\
cgs2014a & 0.025 & -0.065 & 0.018 & 0.469 & 0.134 & 0.141 & 0.071 & 1.74 & 0.153 \\
gsf2014a & 0.033 & -0.087 & 0.115 & -0.102 & 0.131 & 0.129 & 0.067 & 1.28 & 0.147 \\
iaa2007a & 0.027 & -0.065 & 0.583 & -0.501 & 0.138 & 0.145 & 0.083 & 1.60 & 0.143 \\
opa2015a & 0.025 & -0.078 & 0.166 & -0.092 & 0.140 & 0.135 & 0.060 & 1.40 & 0.180 \\
spu00004 & 0.072 & -0.105 & 0.058 & -0.714 & 0.150 & 0.148 & 0.072 & 1.85 & 0.158 \\
usn2015a & 0.026 & -0.078 & 0.128 & -0.054 & 0.130 & 0.128 & 0.069 & 1.23 & 0.139 \\
vieeop13 & -0.005 & -0.065 & 0.135 & 0.067 & 0.148 & 0.150 & 0.080 & 1.87 & 0.152 \\
ivs14q2X & 0.048 & -0.076 & 0.073 & -0.197 & 0.119 & 0.124 & 0.098 & 0.84 & 0.109 \\
eop08c04 & 0.041 & -0.084 & 0.131 & -0.008 & 0.141 & 0.150 & 0.077 & 1.24 & 0.162 \\
eopbullA & -0.026 & -0.093 & 0.424 & -0.452 & 0.166 & 0.157 & 0.067 & 1.08 & 0.218 \\
\hline
\end{tabular}



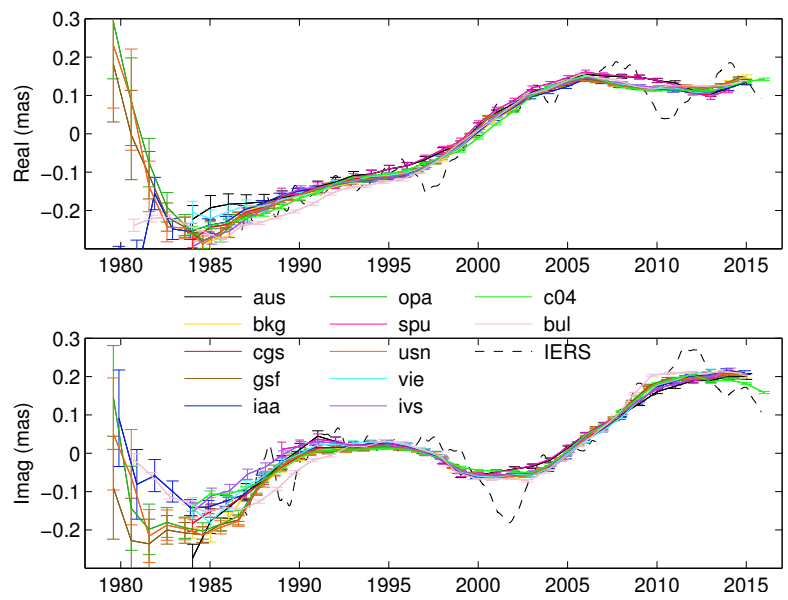

Fig. 2 (Top) Real and (Bottom) imaginary parts of the amplitude of the annual values of the FCN amplitude obtained from the analysis of the nutation time series. The color code follows the Fig. 1. 
Gattano et al.

Table 3 Median amplitudes of the correction to IAU 2000A adjusted to the nutation time series, highest difference between values (Range), and median standard deviation. Unit is $\mu$ as.

\begin{tabular}{|c|c|c|c|c|c|c|}
\hline \multirow{2}{*}{$\begin{array}{l}\text { Period } \\
\text { (days) }\end{array}$} & \multicolumn{2}{|c|}{ Median amplitude } & \multicolumn{2}{|c|}{ Range } & \multicolumn{2}{|c|}{ Median errol } \\
\hline & Real & Imag & Real & Imag & Real & Imag \\
\hline-6798.38 & 35.5 & -28.7 & 47.4 & 17.6 & 2.2 & 2.2 \\
\hline 6798.38 & 22.4 & -32.8 & 18.9 & 18.3 & 2.2 & 2.2 \\
\hline-3399.19 & 4.5 & -9.4 & 15.8 & 11.7 & 2.1 & 2.1 \\
\hline 3399.19 & 11.3 & -5.2 & 10.8 & 24.0 & 2.1 & 2.1 \\
\hline-1615.75 & -2.0 & -8.1 & 17.8 & 9.3 & 2.1 & 2.1 \\
\hline 1615.75 & 0.9 & -9.1 & 12.2 & 6.7 & 2.1 & 2.1 \\
\hline-1305.48 & 0.6 & 8.2 & 13.4 & 10.0 & 2.1 & 2.1 \\
\hline 1305.48 & 0.0 & 4.4 & 11.8 & 12.8 & 2.1 & 2.1 \\
\hline-1095.18 & -1.1 & 1.1 & 10.7 & 10.5 & 2.0 & 2.0 \\
\hline 1095.18 & -3.3 & -1.2 & 9.5 & 9.2 & 2.0 & 2.0 \\
\hline-386.00 & -1.2 & -1.4 & 12.5 & 7.7 & 2.0 & 2.0 \\
\hline 386.00 & -4.3 & 1.9 & 10.6 & 11.7 & 2.0 & 2.0 \\
\hline-365.26 & 27.5 & 5.6 & 9.4 & 18.6 & 2.1 & 2.1 \\
\hline 365.26 & -3.8 & -0.9 & 15.8 & 21.6 & 2.1 & 2.1 \\
\hline-346.64 & -13.3 & 4.7 & 16.6 & 10.6 & 2.2 & 2.2 \\
\hline 346.64 & -2.2 & 2.2 & 6.8 & 8.7 & 2.2 & 2.2 \\
\hline-182.62 & -15.2 & 4.2 & 8.5 & 6.6 & 2.0 & 2.0 \\
\hline 182.62 & 7.5 & -2.8 & 14.0 & 12.5 & 2.0 & 2.0 \\
\hline-121.75 & -3.7 & 2.2 & 4.8 & 5.6 & 2.0 & 2.0 \\
\hline 121.75 & 3.1 & -0.2 & 6.4 & 10.8 & 2.0 & 2.0 \\
\hline-31.81 & -1.0 & -2.7 & 9.5 & 6.2 & 2.0 & 2.0 \\
\hline 31.81 & -1.4 & 1.4 & 6.0 & 6.4 & 2.0 & 2.0 \\
\hline-27.55 & -16.2 & -8.3 & 8.3 & 6.9 & 2.0 & 2.0 \\
\hline 27.55 & -0.7 & -1.6 & 5.3 & 5.3 & 2.0 & 2.0 \\
\hline-23.94 & -2.2 & 0.3 & 3.6 & 3.3 & 2.0 & 2.0 \\
\hline 23.94 & -2.4 & -1.3 & 7.3 & 3.7 & 2.0 & 2.0 \\
\hline-14.77 & -2.3 & 3.5 & 9.8 & 6.6 & 2.0 & 2.0 \\
\hline 14.77 & -1.6 & 0.4 & 3.4 & 6.6 & 2.0 & 2.0 \\
\hline-13.78 & -0.6 & -1.6 & 4.4 & 8.9 & 2.0 & 2.0 \\
\hline 13.78 & -0.6 & 0.1 & 4.0 & 10.2 & 2.0 & 2.0 \\
\hline-13.66 & -11.2 & -10.0 & 10.9 & 7.1 & 2.0 & 2.0 \\
\hline 13.66 & -6.0 & 11.6 & 17.4 & 22.6 & 2.0 & 2.0 \\
\hline-9.56 & 0.6 & -0.9 & 5.4 & 3.0 & 2.0 & 2.0 \\
\hline 9.56 & 1.1 & -0.8 & 7.0 & 8.5 & 2.0 & 2.0 \\
\hline-9.13 & -3.5 & -0.1 & 6.0 & 4.8 & 2.0 & 2.0 \\
\hline 9.13 & -1.5 & 2.7 & 5.7 & 14.3 & 2.0 & 2.0 \\
\hline-9.12 & 1.1 & 0.8 & 9.5 & 9.3 & 2.0 & 2.0 \\
\hline 9.12 & -0.5 & -1.4 & 8.3 & 12.1 & 2.0 & 2.0 \\
\hline-7.10 & -1.8 & -1.0 & 6.2 & 8.8 & 2.0 & 2.0 \\
\hline 7.10 & -2.8 & 6.5 & 11.8 & 10.7 & 2.0 & 2.0 \\
\hline-6.86 & -2.6 & -2.5 & 7.3 & 13.1 & 2.2 & 2.2 \\
\hline 6.86 & 1.8 & -0.4 & 6.9 & 5.7 & 2.2 & 2.2 \\
\hline
\end{tabular}



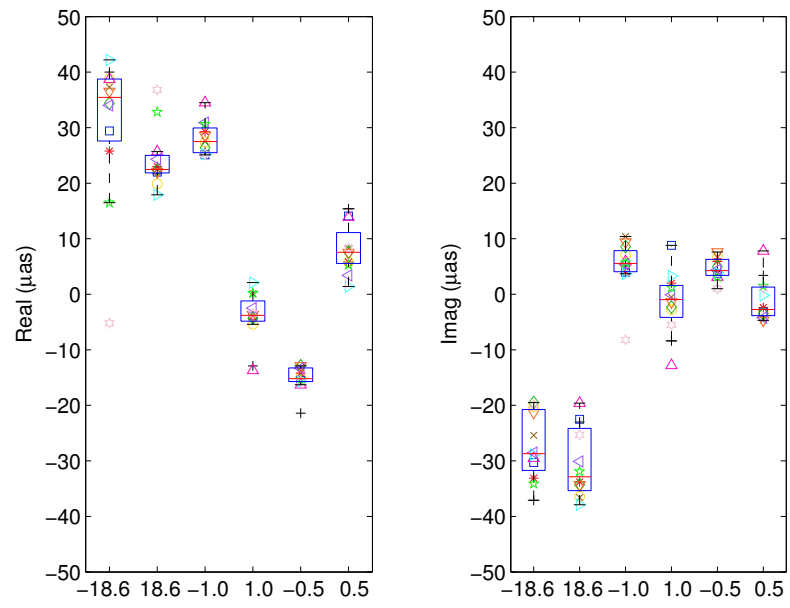

Period (yr)
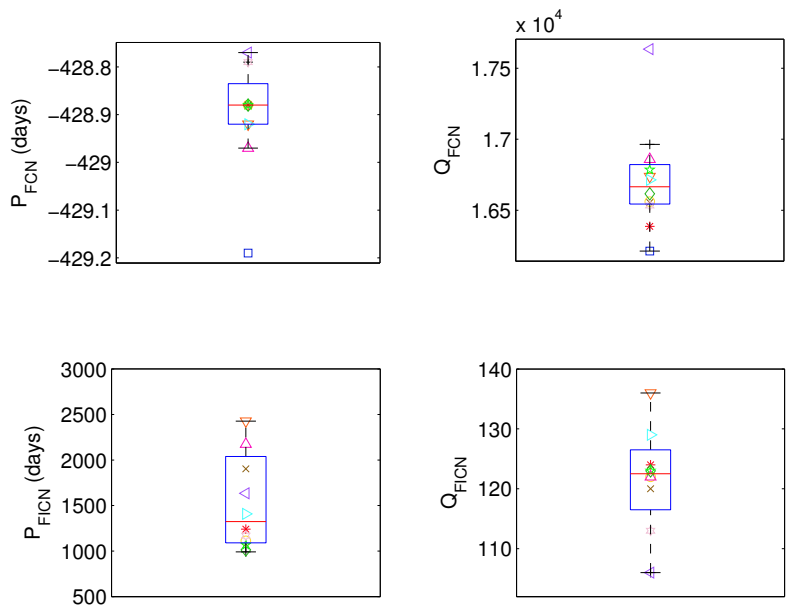

Fig. 3 Top panels: estimated corrections to the IAU 2000A amplitudes of the \pm 18.6 , annual, and semi-annual nutations. Bottom panels: periods and quality factors of the FCN and FICN estimated from previous nutation amplitudes. The size of the whiskers corresponds to the standard deviation of the estimates from the various solutions. The horizontal red line represents the median value. The color code follows Fig. 1. Plus sign: aus00007; Circle: bkg00014; Asterisk: cgs2014a; Cross: gsf2014a; Square: iaa2007a; Diamond: opa2015a; Upward-pointing triangle: spu00004; Downward-pointing triangle: usn2015a; Right-pointing triangle: vieeop13; Left-pointing triangle: ivs14q2X; Pentagram: eop08c04; Hexagram: eopbullA. 